\title{
Challenges and Considerations for Delivering Bioinformatics Training in LMICs: Perspectives From Pan-African and Latin American Bioinformatics Networks
}

\section{OPEN ACCESS}

Edited by:

Hugo Verli,

Federal University of Rio Grande do

Sul, Brazil

Reviewed by:

Laurent Emmanuel Dardenne, National Laboratory for Scientific

Computing (LNCC), Brazil

Ana Carolina Guimarães, Oswaldo Cruz Institute(FIOCRUZ),

Brazil

*Correspondence:

Marco Cristancho

ma.cristancho29@uniandes.edu.co

Specialty section:

This article was submitted to

STEM Education,

a section of the journal

Frontiers in Education

Received: 17 May 2021 Accepted: 12 July 2021 Published: 27 July 2021

Citation:

Ras V, Carvajal-López $P$,

Gopalasingam $P$, Matimba $A$,

Chauke PA, Mulder N, Guerfali $F$,

Del Angel VD, Reyes A, Oliveira G,

De Las Rivas $J$ and Cristancho $M$ (2021) Challenges and Considerations for Delivering Bioinformatics Training in LMICs: Perspectives From Pan-African and Latin American

Bioinformatics Networks.

Front. Educ. 6:710971.

doi: 10.3389/feduc.2021.710971

\begin{abstract}
Verena Ras ${ }^{1}$, Patricia Carvajal-López ${ }^{2}$, Piraveen Gopalasingam ${ }^{2}$, Alice Matimba ${ }^{2}$, Paballo Abel Chauke ${ }^{1}$, Nicola Mulder ${ }^{1}$, Fatma Guerfali ${ }^{3}$, Victoria Dominguez Del Angel ${ }^{4}$, Alejandro Reyes ${ }^{5}$, Guilherme Oliveira ${ }^{6}$, Javier De Las Rivas ${ }^{7}$ and Marco Cristancho ${ }^{8 *}$

${ }^{1}$ Computational Biology Division, Department of Integrative Biomedical Sciences, Institute of Infectious Disease and Molecular Medicine, CIDRI Africa Wellcome Trust Centre, Faculty of Health Sciences, University of Cape Town, Cape Town, South Africa, ${ }^{2}$ European Molecular Biology Laboratory, European Bioinformatics Institute, Hinxton, United Kingdom, ${ }^{3}$ Institut Pasteur de Tunis, Tunis, Tunisia, ${ }^{4}$ INRAe, Centre de Recherche de Versailles, Institut Français de Bioinformatique, UMS3601-CNRS, Paris, France, ${ }^{5}$ Max Planck Tandem Group in Computational Biology, Department of Biological Sciences, Universidad de Los Andes, Bogotá, Colombia, ${ }^{6}$ Instituto Tecnologico Vale, Belem, Brazil, ${ }^{7}$ Cancer Research Center (CiC-IBMCC), Consejo Superior de Investigaciones Cientificas (CSIC) and Universidad de Salamanca (USAL), Campus Miguel de Unamuno s/n, Salamanca, Spain, ${ }^{8}$ Vicerrectoria de Investigación y Creación, Universidad de Los Andes, Bogotá, Colombia
\end{abstract}

Keywords: training, low to middle income countries, best practices, Africa, Latin America

\section{INTRODUCTION}

In general, institutions and research groups based in Low to Middle Income Countries (LMICs) battle a number of challenges ranging from a shortage of infrastructure, lack of training facilities, and poor internet, to a lack of local expertise (Karikari et al., 2015; Tastan Bishop et al., 2015; Shaffer et al., 2019). A shortage of local experts, particularly within more specialised topics, still remains a key obstacle in developing bioinformatics research capacity within LMICs (Tastan Bishop et al., 2015; Mulder et al., 2018).

Over the last decade, some barriers experienced by scientists in LMICs have been lowered, in part, by increasing access to more reliable internet, facilities and infrastructure (De Las Rivas et al., 2019; Mulder and H3ABioNet, 2020). This has facilitated the analysis of increasingly complex datasets, which has led to a growing deficit in scientists able to perform these analyses. Access to high quality, affordable training resources and well-trained trainers is typically also a major challenge. Training within LMICs, similar to any context, requires a well-planned approach and often depends on a unique understanding of their specific challenges in order to provide effective training. It is thus useful to outline what we consider to be a range of best practices that may be followed when designing and delivering training in LMICs that may significantly increase a training event's chance of success and lasting impact. We focus here on short term training (workshops, hackathons or data analysis jamborees, etc.) but a number of the best practices provided can be broadly applied to degree modules-in both undergraduate and postgraduate programmes (Fatumo et al., 2014).

We have curated a set of Best Practices for delivering Bioinformatics Training in LMICs, with an assemblage of guidelines available as a "living document" on GitHub. The "living document" is based on our own experiences in different settings, where we aim to highlight some of the major challenges that organizers and trainers might face when delivering bioinformatics training in LMICs. These challenges have been divided into three key categories/themes i.e., 1) Planning, 2) Development and 
3) Implementation, which loosely represent the three major phases of organizing and delivering a training event, and provides some potential solutions, guidelines or reusable templates.

\section{PLANNING}

Training should be tailored for relevance and application to topics of interest in the country or region where the training is taking place rather than a broad bioinformatics subject. Given the particular locations and interests of LMICs, some of the subjects that might be of great interest include biodiversity, rare and communicable diseases, tropical diseases, and native crops research. It is always possible to incorporate social aspects in technical subjects such as genomics and bioinformatics and organizers should make an effort to achieve that as a goal for all training events.

A key challenge in LMICs, even where knowledge, skills and capacity are available locally, is that there may be limited financial or institutional support. Organizers require buy-in and commitment from key stakeholders to support training development and delivery. Even a small/short course may require close involvement of the heads of institutions, government ministries and political representatives who can influence decisions that provide money and infrastructure. The involvement of public agencies will also have the benefit of contextualising the training to local and regional socioeconomic environments.

Unlike in High Income Countries (HICs), well-renowned institutions, societies and even local governments might play a significant role in the support and organization of training activities in LMICs, even in such a technical subject as bioinformatics. This is very convenient for funding of the event, infrastructure, involvement of public institutions and even the inclusion of trainers who have been involved in policy making and other global treaties. For instance, training organizers and scientists can influence incorporation of relevant training in programmes aimed at building capacity for biodiversity. Equally, training on use of human data should include information about local data sharing policies and ethics.

Regionally, LMICs show variable degrees of development in bioinformatics training. For instance Brazil, Chile, Argentina, México and Colombia have relatively advanced undergraduate and postgraduate bioinformatics programs compared to other countries in Latin America, which are lagging behind in capacity for delivering training (De Las Rivas et al., 2019). Therefore it is imperative to collaborate with scientists from countries which need support for establishing training initiatives. This is particularly important to reinforce the United Nations' Sustainable Development Goals (SDGs, 2015) where inclusive and equitable education is key to reaching both gender and race equity, globally.

Having explored the above issues and once training goals have been set, a project plan is useful for effective implementation of the training. An ideal development team should map out the tasks required to effectively deliver the training on time and within a proposed budget. Training or scientific development involves leadership in the development of resources, setting the timelines and developing the training agenda. Event administration is important for overall logistical organization and processes. Technical expertise is required for bioinformatics training for ensuring infrastructure, training resources and softwares are prepared and functional. Tasks such as travel arrangements can be outsourced if needed but can be dealt with "in-house" where enough logistical expertise or guidance is available.

\section{DEVELOPMENT}

A number of training organizations provide information about their courses and often even provide access to the training materials from their courses. It is good to explore what these organizations are doing successfully and if appropriate, reach out for advice, guidance or support in the form of collaborative funding or delivery. H3ABioNet for example, administers a free to use public helpdesk (https://helpdesk.h3abionet.org/) where a variety of queries can be submitted. One could look at the models being used, content focused on and determine whether any of their approaches may work for your purposes. CABANA is also developing e-learning resources relevant for Latin American research scientists and this material will be available in Spanish (https://www.cabana.online/elearning). Some organizations and conference organizers partner with regional teams to develop workshops linked to conferences offering value addition to attendees and maximizing on the convergence of a specific target audience for scientific topics.

Securing the most appropriate instructors is important and has implications for funding and training format. When holding a regional course in LMICs, where international trainers are involved, the input of local scientists and experts should be a primary consideration. Firstly, local instructors have a sound appreciation of the target audience, they understand local research interests and needs and are comfortable with the local languages/dialects. Secondly, local scientists have the unique opportunity to strengthen and grow local capacity for bioinformatics training by collaborating and, working with guest trainers or international experts, who typically have scarce or special skills, thus increasing sustainability for training and research locally. It is important to acknowledge that representation matters, especially along gender, ethnicity, expertise, and geography. A diverse trainer team can make your course look more attractive, encouraging higher registrations.

It is vital that course organizers provide support, resources, and guidance to course developers/contributors/instructors throughout the development process. It is often useful to conceptualize your training first using a brainstorming document or perhaps a planning template like the one developed by $\mathrm{H} 3 \mathrm{ABioNet}$ (this and other templates can be accessed and downloaded at this link).

Language can be a major barrier where trainees are not fluent in the language of the event. An option is to provide language support during the course by having instructors or additional 


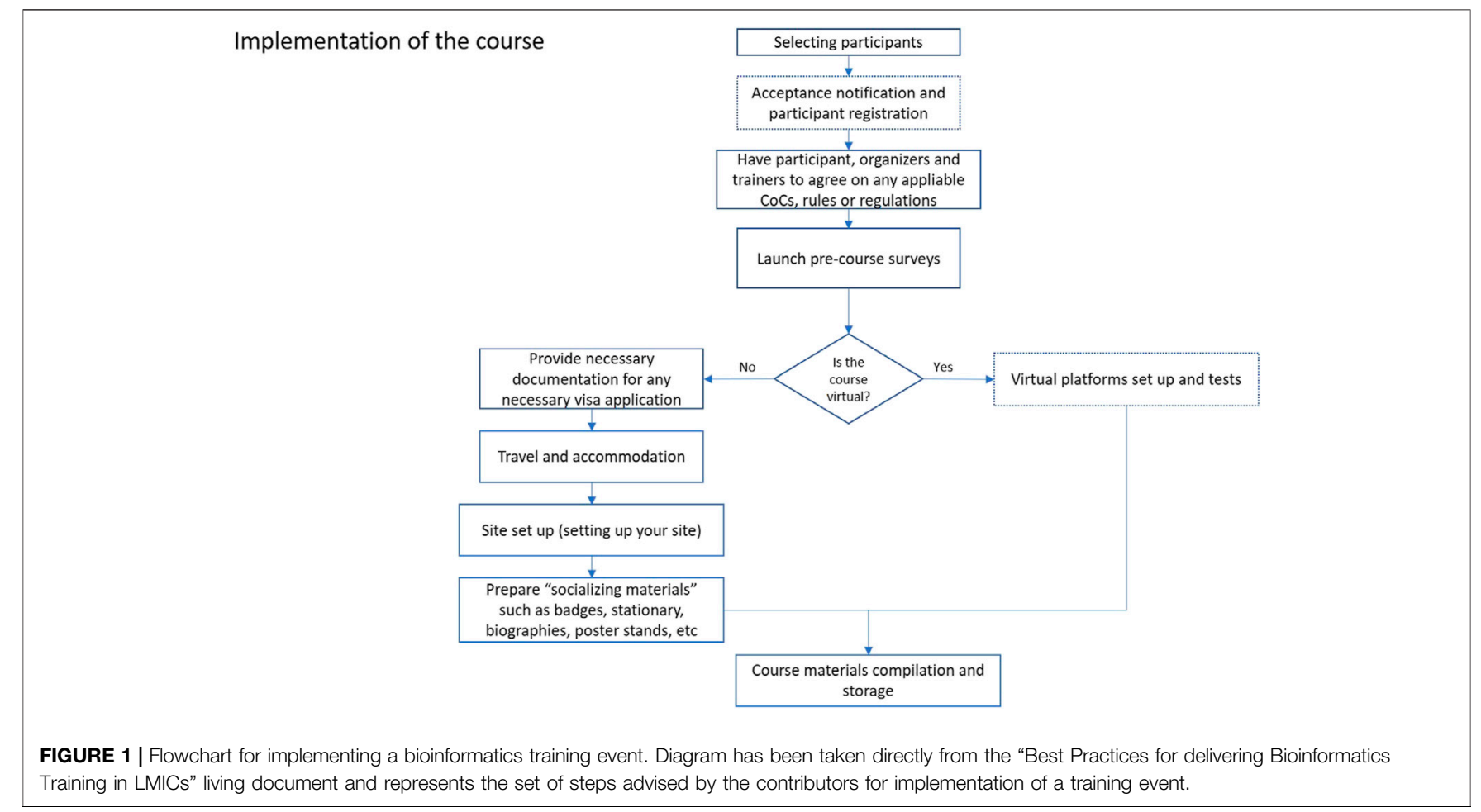

assistants who speak the local language. It is highly recommended to provide introductory courses in the local language of the trainees; introducing complex topics such as genomics and bioinformatics. It is recommended to reduce costs, to use reference materials such as articles, books and databases which have open access licenses.

\section{IMPLEMENTATION}

In Figure 1 we describe the main steps that must be followed for implementation of a workshop/course. Selection of participants should strongly consider gender and geographical diversity, ensuring that training can reach diverse participants in the region. A key challenge for face-to-face courses for travelling participants however, is the visa application process - this step can be one of the lengthiest in the whole process of organizing a course. For trainees, a visitor visa is usually sufficient for participating in the workshop/course; for trainers some institutions require a work visa, even for short training courses. Venues for training events must preferably be chosen within close proximity to the accommodation of trainers and trainees. Transportation in most large and middle-size cities can be rather problematic and public transport might not be the best option due to issues like safety and long commute times.

A final consideration is the socio-political situation that must be taken into account when organizing any training in LMICs. In some LMICs civil unrest, political and economic instability, means that safety and infrastructure circumstances may change over short periods of time. It is advisable for organizers to insure any costs that might be reimbursable in case the training event has to be postponed or cancelled. Contingency plans should be made for running events with less infrastructural capacity, such as having pre-recorded content or non-computer-based exercises. Offline solutions like the eBioKit (http://www.ebiokit.eu/) should also be considered in regions with unreliable internet access.

\section{CONCLUDING REMARKS}

It is noted by the authors of this piece, that the advent of blended/ online learning approaches holds the potential to resolve many of the points raised here. Some general guidelines for virtual bioinformatics teaching have been published given the recent Covid-19 pandemic world lockdown (Gallardo-Alba et al., 2021). H3ABioNet likewise, have developed a comprehensive "multipledelivery-approach training model" that combines both physical face-to-face style classrooms (situated in any country) with online learning (using a Learning Management System to manage trainees/local staff), distance learning (trainers connect from anywhere in the world to participants and staff in distributed classrooms) and open educational resources (OER) (all materials developed under a creative commons [cc-by] license and publicly accessible). This model has helped H3ABioNet reach upwards of 1,000 participants annually (see Ras et al., 2021). While H3ABioNet has extended this model to deliver more advanced, data intensive training, it must be noted that although blended and online learning can result in higher participation/uptake of the training, there are still many limitations. Highly advanced/technical topics are still difficult to teach as participants or institutions may lack ongoing local 
capacity or support. It is also the experience of all authors that the practical/hands-on components of highly advanced topics become increasingly difficult to teach well in an online or hybrid environment, while skills transfer between trainers, trainees and (potential) international trainers is typically greatly reduced. Thus, there currently is no complete substitute for face-to-face/hands-on workshops.

Bioinformatics training in LMICs have a lasting positive impact if done well, ranging from individual and institutional capacity development to better research, more collaboration, and increased funding for activities. Training organizers and developers should tailor content, format and style to suit the local or regional needs and environment. This can be strengthened by community building efforts during and after training events which supports building and maintaining a critical mass of skilled scientists.

A vital consideration that anyone endeavouring to deliver bioinformatics training must at all times keep in mind, is availability of materials, resources and tools post-course. Some LMICs have enhanced local infrastructure, however, many institutions and regions exist where infrastructure has not progressed. This means trainees may not have access to appropriate resources and tools after a course ends to perform real analyses. This perhaps remains one of the most challenging obstacles to overcome but designing a course with this in mind often leads to a more well-received course and may mitigate some of the infrastructural challenges. It often also fosters creativity and solution-driven thinking locally. Finally, please refer to our "Best Practices for delivering Bioinformatics Training in LMICs (link)" living document, for the perspectives that we have highlighted in

\section{REFERENCES}

De Las Rivas, J., Bonavides-Martínez, C., and Campos-Laborie, F. J. (2019). Bioinformatics in Latin America and SolBio Impact, a Tale of Spin-Off and Expansion Around Genomes and Protein Structures. Brief. Bioinform. 20 (2), 390-397. doi:10.1093/bib/bbx064

Fatumo, S., Shome, S., and Macintyre, G. (2014). Workshops: A Great Way to Enhance and Supplement a Degree. Plos Comput. Biol. 10 (2), e1003497. doi:10.1371/journal.pcbi.1003497

Gallardo-Alba, C., Grüning, B., and Serrano-Solano, B. (2021). A ConstructivistBased Proposal for Bioinformatics Teaching Practices during Lockdown. Plos Comput. Biol. 17 (5), e1008922. doi:10.1371/journal.pcbi.1008922

Karikari, T. K., Quansah, E., and Mohamed, W. M. Y. (2015). Developing Expertise in Bioinformatics for Biomedical Research in Africa. Appl. Translational Genomics 6, 31-34. doi:10.1016/j.atg.2015.10.002

Mulder, N., Abimiku, A. L., Adebamowo, S. N., de Vries, J., Matimba, A., Olowoyo, P., et al. (2018). H3Africa: Current Perspectives. Pharmgenomics Pers Med. 11, 59-66. doi:10.2147/PGPM.S141546

Mulder, N. J., and H3ABioNet (2020). H3ABioNet, Infrastructure for African Genomics Data. Gates Open Res. 4, 132. doi:10.21955/gatesopenres.1116670.1

Ras, V., Botha, G., Aron, S., Lennard, K., Allali, I., Claassen-Weitz, S., et al. (2021). Using a Multiple-Delivery-Mode Training Approach to Develop Local Capacity and Infrastructure for Advanced Bioinformatics in Africa. Plos Comput. Biol. 17 (2), e1008640. doi:10.1371/ journal.pcbi.1008640

SDGs, U. N. (2015). United Nations Sustainable Development Goals. Available at: https://sdgs.un.org/goals (Accessed March 1, 2021). this opinion article and to broaden the guidelines that we suggest you consider when delivering bioinformatics training in LMICs.

\section{AUTHOR CONTRIBUTIONS}

All authors listed have made a substantial, direct, and intellectual contribution to the work and approved it for publication.

\section{FUNDING}

The authors who contributed to this manuscript are funded in whole, or in part, by the Wellcome Trust (WT108749/Z/15/ Z), (WT108749/Z/15/A), H3ABioNet NIH grant U24HG006941, H3ABioNet NIH grant U24HG006942, CABANA-Capacity building for bioinformatics in Latin America' (CABANA), funded by UKRI-BBSRC on behalf of the Global Challenges Research Fund (BB/P027849/1), CSIC/ USAL and EMBL-EBI.

\section{ACKNOWLEDGMENTS}

The authors would like to acknowledge the many contributors of templates, case studies and experiences to the larger body of work contained on Github and who may not necessarily be authors on this particular paper. A full list of contributors to the larger project can be accessed on the Github repository (https://bioinfotraining.github.io/guidelines/).

Shaffer, J. G., Mather, F. J., Wele, M., Li, J., Tangara, C. O., Kassogue, Y., et al. (2019). Expanding Research Capacity in Sub-saharan Africa through Informatics, Bioinformatics, and Data Science Training Programs in Mali. Front. Genet. 10, 331. doi:10.3389/fgene.2019.00331

Tastan Bishop, O., Adebiyi, E. F., Alzohairy, A. M., Everett, D., Ghedira, K., Ghouila, A., et al. (2015). Bioinformatics Education-Pperspectives and Challenges Out of Africa. Brief. Bioinform. 16 (2), 355-364. doi:10.1093/bib/ bbu022

Conflict of Interest: The authors declare that Patricia Carvajal-López is a coordinator of the Original Strategies for Training and Educational Initiatives in Bioinformatics Research Topic. All other authors have declared no further conflicts of interest.

Publisher's Note: All claims expressed in this article are solely those of the authors and do not necessarily represent those of their affiliated organizations, or those of the publisher, the editors and the reviewers. Any product that may be evaluated in this article, or claim that may be made by its manufacturer, is not guaranteed or endorsed by the publisher.

Copyright (c) 2021 Ras, Carvajal-López, Gopalasingam, Matimba, Chauke, Mulder, Guerfali, Del Angel, Reyes, Oliveira, De Las Rivas and Cristancho. This is an openaccess article distributed under the terms of the Creative Commons Attribution License (CC BY). The use, distribution or reproduction in other forums is permitted, provided the original author(s) and the copyright owner(s) are credited and that the original publication in this journal is cited, in accordance with accepted academic practice. No use, distribution or reproduction is permitted which does not comply with these terms. 